\title{
The prognostic significance of co-existence ductal carcinoma in situ in invasive ductal breast cancer: a large population-based study and a matched case-control analysis
}

\author{
Hongliang Chen, Fang Bai, Maoli Wang, Mingdi Zhang, Peng Zhang, Kejin Wu \\ Department of Breast Surgery, Obstetrics and Gynecology Hospital of Fudan University, Shanghai 200011, China \\ Contributions: (I) Conception and design: H Chen, K Wu; (II) Administrative support: H Chen, K Wu; (III) Provision of study materials or patients: \\ H Chen, F Bai; (IV) Collection and assembly of data: H Chen, F Bai, M Wang; (V) Data analysis and interpretation: H Chen, F Bai, M Wang; (VI) \\ Manuscript writing: All authors; (VII) Final approval of manuscript: All authors. \\ Correspondence to: Kejin Wu. Department of Breast Surgery, Obstetrics and Gynecology Hospital of Fudan University, 419 Fangxie Road, Shanghai \\ 200011, China. Email: kejinwu1128@163.com.
}

Background: To evaluate the prognostic significance of co-existence ductal carcinoma in situ (DCIS) in
invasive ductal breast cancer (IDC) compared with pure IDC.
Methods: The Surveillance, Epidemiology, and End Results (SEER) database was searched to identify
unilateral IDC cases between 2004 and 2015, which were grouped into pure IDC and IDC with DCIS
component (IDC-DCIS). Comparisons of the distribution of clinical-pathological characteristics the two
groups were performed using Pearson's chi-square. Breast cancer-specific survival (BCSS) and overall survival
(OS) were estimated using the Kaplan-Meier method and compared across RS groups using the log-rank
statistic. Cox models were fitted to assess the factors independently associated with survival. A $1: 1$ matched
case-control analysis was conducted with each clinical-pathological characteristic matched completely.
Results: A total of 98,097 pure IDC cases ( $39.6 \%)$ and 149,477 IDC-DCIS cases (60.4\%) were enrolled.
IDC-DCIS patients were presented with less aggressive characteristics such as lower proportion of histologic
grade III ( $34.2 \%$ vs. $42.2 \%$, P<0.001), ER negative ( $16.8 \%$ vs. $26.1 \%$, P<0.001) and PR negative ( $26.5 \%$
vs. $35.7 \%$, P<0.001) disease and higher proportion of T1 cases ( $68.7 \%$ vs. $58.2 \%$, P $<0.001)$ compared with
pure IDC patients. Co-existence DCIS was an independent prognostic factor for BCSS and OS in the whole
cohort. According to the multivariate analysis, it was an independent favorable prognostic factor among ER
positive cases, but an independent negative prognostic factor among ER negative cases based on the matched
cohort.

Conclusions: Co-existence DCIS showed quite different prognostic significance among ER positive and negative disease.

Keywords: Invasive ductal breast cancer (IDC); ductal carcinoma in situ (DCIS); co-existence; prognosis; population-based; case-control

Submitted Apr 07, 2019. Accepted for publication Jul 09, 2019.

doi: $10.21037 /$ atm.2019.08.16

View this article at: http://dx.doi.org/10.21037/atm.2019.08.16

\section{Introduction}

Ductal carcinoma in situ (DCIS) is a proliferation of malignant cells which do not invade the basement membrane of the breast ducts. DCIS is a nonobligate precursor to infiltrating ductal carcinoma of the breast
(IDC). A substantial proportion of patients with IDC have accompanying DCIS component. It was reported that the percentage of cases with DCIS associated with invasive cancer varied significantly from $21.3 \%$ to $76.9 \%$ in the literature (1-6). 
Although some studies have investigated the clinicalpathological characteristics of DCIS that are associated with invasive disease, the role of concomitant DCIS on the prognosis of patients with IDC has not been well studied in the literature. Currently in the TNM staging system $\mathrm{T}$ category is based on the size of the invasive component alone. The association of DCIS in IDC has no bearing on systemic treatment, which depends entirely on the pathological and molecular characteristics of IDC. Different distribution of subtypes and distinctive characteristics among DCIS, DCIS accompanying microinvasive cancer, and IDC accompanying DCIS (IDC-DCIS) indicate that IDC-DCIS represents a disease entity distinct from pure IDC (7). In fact, some researches showed that IDC with accompanying DCIS tended to have a favorable biology and survival outcome in spite of no statistical significance $(2,8-10)$ while opposite results have also been demonstrated (4). The related studies involved only a small sample size, and the clinical significance associated with accompanying DCIS in invasive disease has not been conclusively defined.

The purpose of the present study was to evaluate the prognostic value of concomitant DCIS component in a large cohort of patients with invasive ductal breast cancer (IDC) based on the Surveillance, Epidemiology, and End Results (SEER) 18 database.

\section{Methods}

\section{Patient population}

This population-based study used data derived from the National Cancer Institute's limited use SEER 18 registry databases released in November 2018. We identified unilateral primary IDC between 2004 and 2015. Using the collaborative stage data set coding, pure IDC group was defined as entire tumor reported as IDC without DCIS component. Meanwhile, IDC-DCIS group was defined as coexistence of IDC and DCIS components, and the T category was based primarily on the size of the invasive component of the cancer. Cases in which tumor size was coded both IDC and DCIS components due to size of IDC component not stated were excluded. Besides, the study also excluded patients with more than one primary cancer, diagnosis at death or autopsy alone, T0, Tis, T1mic disease, unknown $\mathrm{T}$ or $\mathrm{N}$ category, no surgery performed or no record of surgery. TNM stage was based on a derived
AJCC 6th edition [2004-2009] and 7th edition [2010-2015]. Poorly differentiated and anaplastic histologic grades were considered grade III disease. Borderline status of ER or PR was defined as unknown. We obtained permission to access the files of SEER program custom data with additional treatment fields such as radiation therapy and chemotherapy. Informed consent was not required because personal identifying information was not involved. This study was reviewed and approved by the Institutional Review Board of Obstetrics and Gynecology Hospital of Fudan University.

\section{Statistical analysis}

The distributions of clinical-pathological characteristics according to accompanying DCIS status were compared using Pearson's Chi square with Fisher's exact test. The follow-up cutoff was 31 December 2016. Breast cancerspecific survival (BCSS) was computed from the time of breast cancer diagnosis to the time of death from breast cancer or the last follow-up evaluation with patients still alive at the last censored follow-up. Overall survival (OS) was computed from the time of diagnosis to the time of death from any cause or the last follow-up evaluation with patients still alive at the last censored follow-up. Both BCSS and OS were estimated using the Kaplan-Meier method and compared across concomitant DCIS status using the log-rank statistic. Adjusted hazard ratios (HRs) with $95 \%$ confidence intervals (CIs) were calculated using the Cox model to identify significant independent factors associated with survival. To diminish the effects of baseline differences on outcome differences in the IDC-DCIS group and pure IDC group, the propensity score matching method was applied by matching each pure IDC case to one IDCDCIS case. They were exactly matched for year of diagnosis ( \pm 2 years), age stage $(<40,40-49,50-59,60-69,70-79$, and $\geq 80$ years), race, histologic grade, $T$ and $\mathrm{N}$ category, ER and PR status, HER2 status (since 2010), surgery type, radiation and chemotherapy. As over $90 \%$ cases were in $\mathrm{T} 1$ and $\mathrm{T} 2$, T1 category was matched further based on T1a, T1b, T1c, and T2 category were matched further based on tumor size $\leq 3, \leq 4$ and $\leq 5 \mathrm{~cm}$. A two-sided $\mathrm{P}$ value lower than 0.05 was considered statistically significant. All the statistical analyses were performed using the SPSS 22.0 software package (SPSS, Chicago, IL, USA). 


\section{Results}

Comparison of clinical-pathological characteristics between patient with pure IDC and IDC-DCIS

A total of 98,097 pure IDC cases (39.6\%) and 149,477 IDCDCIS cases $(60.4 \%)$ who met the inclusion criteria between 2004 and 2015 were enrolled in this study. The median age of the whole cohort was 59 years (15-108 years). Most patients were of White race $(78.7 \%)$ and in earlier stage (53.8\% in AJCC stage I, $64.5 \%$ in T1 and $69.3 \%$ in N0). Besides, most patients had ER positive (77.0\%), PR positive $(66.7 \%)$ disease and underwent breast conserving surgery (BCS) (61.1\%). The baseline characteristics of the whole cohort were summarized in Table 1.

Compared with pure IDC patients, those with IDCDCIS were younger (mean age, 58.7 vs. 60.4 years, $\mathrm{P}<0.001$ ) and had more cases under the age of 60 years $(53.4 \%$ vs. $48.3 \%, \mathrm{P}<0.001)$. IDC-DCIS patients were presented with less aggressive characteristics such as lower proportion of histologic grade III $(34.2 \%$ vs. $42.2 \%, \mathrm{P}<0.001)$, ER negative $(16.8 \%$ vs. $26.1 \%, \mathrm{P}<0.001)$ and $\mathrm{PR}$ negative (26.5\% vs. $35.7 \%, \mathrm{P}<0.001)$ disease and higher proportion of cases in AJCC stage I (57.1\% vs. $48.8 \%, \mathrm{P}<0.001)$, T1 (68.7\% vs. $58.2 \%, \mathrm{P}<0.001)$ and $\mathrm{N} 0(69.9 \%$ vs. $68.4 \%$, $\mathrm{P}<0.001)$ category compared with patients with pure IDC. They also received radiation $(54.6 \%$ vs. $56.3 \%, \mathrm{P}<0.001)$ and chemotherapy $(42.6 \%$ vs. $46.5 \%, \mathrm{P}<0.001)$ less frequently. Furthermore, BCS rate was significantly lower in patients with IDC-DCIS compared with patients with pure IDC (60.2\% vs. $62.4 \%, \mathrm{P}<0.001)$. The comparison between patients with pure IDC and IDC-DCIS was also presented in Table 1.

\section{Survival outcomes among patients with pure IDC and IDC-DCIS in the whole cohort}

The median follow-up period was 61 months (range, 0-155 months). The patients with IDC-DCIS had significantly higher BCSS and OS compared with those with pure IDC $(\mathrm{P}<0.001)$ (Figure $1 A, B)$.

In the univariate analysis, year of diagnosis, age stage, race, grade, T, N, surgery, radiation, chemotherapy, ER status, PR status and existence of DCIS component were correlated with BCSS and OS $(\mathrm{P}<0.001)$. According to the multivariate analysis, existence of DCIS component was an independent favorable prognostic factor for BCSS (HR $=0.917,95 \%$ CI: $0.889-0.945, \mathrm{P}<0.001)$ and OS $(\mathrm{HR}=0.858,95 \% \mathrm{CI}: 0.839-0.8773, \mathrm{P}<0.001)$. For cases diagnosed between 2010 and 2015, for which information of HER2 status was available, existence of DCIS component was still an independent favorable prognostic factor for BCSS (HR $=0.908,95 \%$ CI: 0.861-0.958, $\mathrm{P}<0.001$ ) and OS (HR $=0.865$, 95\% CI: 0.831-0.899, $\mathrm{P}<0.001)$.

\section{Survival analysis among pure IDC and IDC-DCIS in the matched cohort}

A 1:1 matched case-control analysis was conducted due to the great baseline difference between IDC-DCIS and pure IDC (Table 1). Each clinical parameter was matched exactly, such as "year of diagnosis ( \pm 2 years), age stage, histologic grade, tumor size, $\mathrm{N}$ category, ER, PR status, HER2 status (since 2010), breast surgery type, radiation therapy and chemotherapy". Altogether 71,662 pure IDC patients could be exactly matched to 71,662 IDC-DCIS patients. There were 4,431 breast cancer related deaths and 9,601 deaths among IDC-DCIS cohort, and 4,318 breast cancer related deaths and 9,897 deaths among pure IDC cohort. Based on the completely matched analysis, IDC-DCIS had a similar BCSS $(\mathrm{P}=0.269)$ but a slightly higher OS $(\mathrm{P}=0.021)$ compared with pure IDC (Figure $1 C, D$ ). According to the multivariate COX analysis, existence of DCIS component proved to be an independent favorable prognostic factor for OS (HR $=0.966, \mathrm{P}=0.016,95 \%$ CI: 0.939-0.994).

In the subgroup analyses, IDC-DCIS had significantly better survival outcomes in histologic grade I $(\mathrm{P}=0.014$ for BCSS and $\mathrm{P}=0.006$ for $\mathrm{OS})$ and II disease $(\mathrm{P}<0.001$ for OS), while pure IDC had a significantly better BCSS and tendency for an improved OS in histologic grade III disease ( $\mathrm{P}=0.009$ for BCSS and $\mathrm{P}=0.069$ for OS) (Figure 2).

Notably, IDC-DCIS had significantly better survival outcomes compared with pure IDC in ER positive cases ( $\mathrm{P}=0.014$ for $\mathrm{BCSS}$ and $\mathrm{P}<0.001$ for OS), whereas pure IDC had significantly better survival outcomes in ER negative cases $(\mathrm{P}<0.001$ for BCSS and $\mathrm{P}=0.002$ for OS) (Figure 3).

IDC-DCIS and pure IDC had similar BCSS and OS in nearly all $\mathrm{T}$ and $\mathrm{N}$ categories except that pure IDC had a better BCSS compared with IDC-DCIS in T1 $(\mathrm{P}=0.011)$ and $\mathrm{N} 0(\mathrm{P}=0.013)$ category. Furthermore, it was demonstrated that in ER positive cases, IDC-DCIS had significantly or tendency of better survival outcomes in T1T3 disease (BCSS: $\mathrm{P}=0.002$ for $\mathrm{T} 2, \mathrm{P}=0.037$ for $\mathrm{T} 3$; OS: $\mathrm{P}=0.002$ for $\mathrm{T} 1, \mathrm{P}=0.001$ for $\mathrm{T} 2, \mathrm{P}=0.179$ for $\mathrm{T} 3$ ) (Figure 4), and in $\mathrm{N} 0-\mathrm{N} 3$ disease (BCSS: $\mathrm{P}=0.018$ for $\mathrm{N} 1, \mathrm{P}=0.944$ for $\mathrm{N} 2, \mathrm{P}=0.008$ for $\mathrm{N} 3$; OS: $\mathrm{P}<0.001$ for $\mathrm{N} 0, \mathrm{P}=0.026$ for $\mathrm{N} 1$, $\mathrm{P}=0.641$ for $\mathrm{N} 2, \mathrm{P}=0.004$ for N3) (Figure 5). However, in 
Table 1 comparison of baseline characteristics between patients with pure IDC and IDC-DCIS

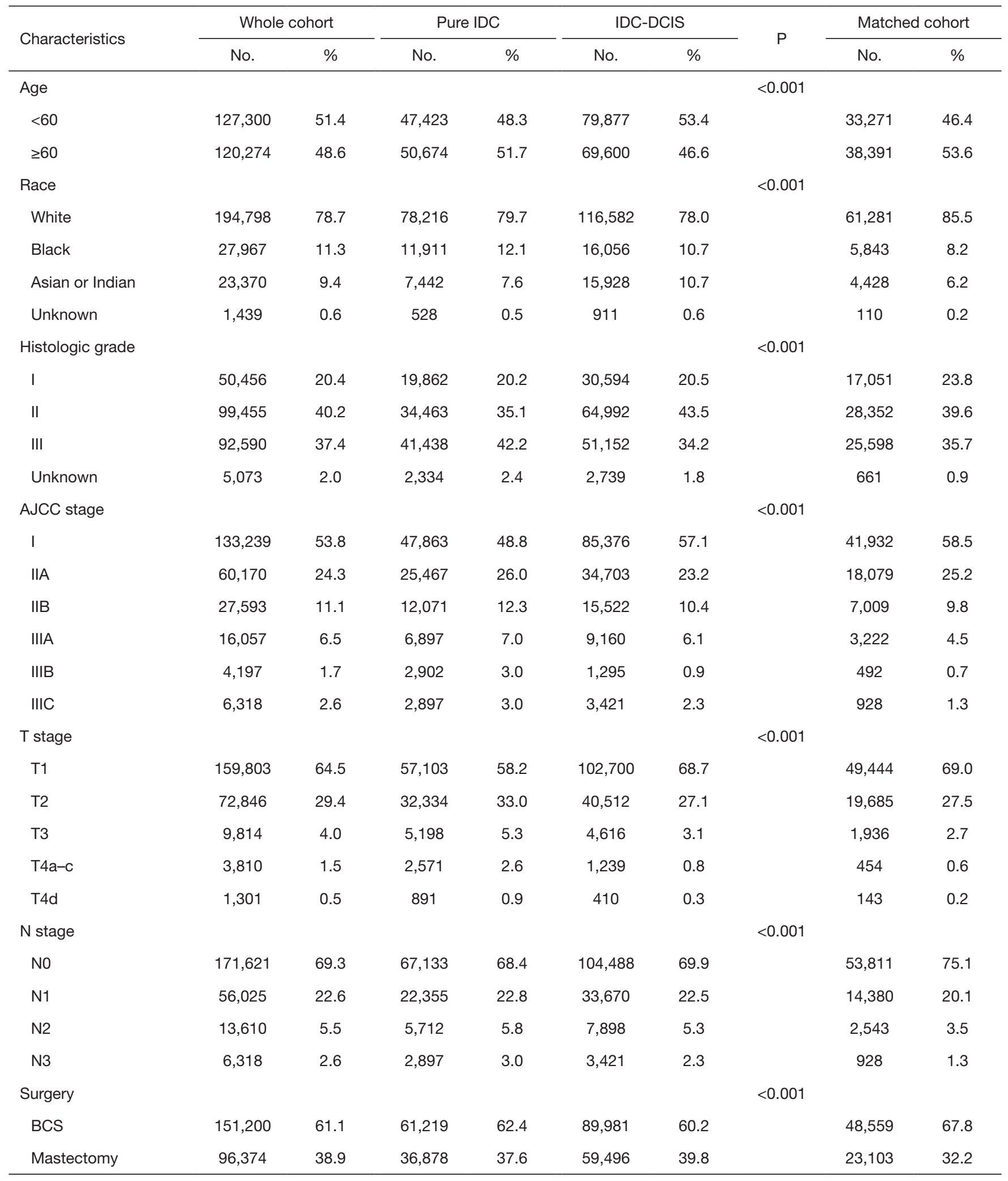

Table 1 (continued) 
Table 1 (continued)

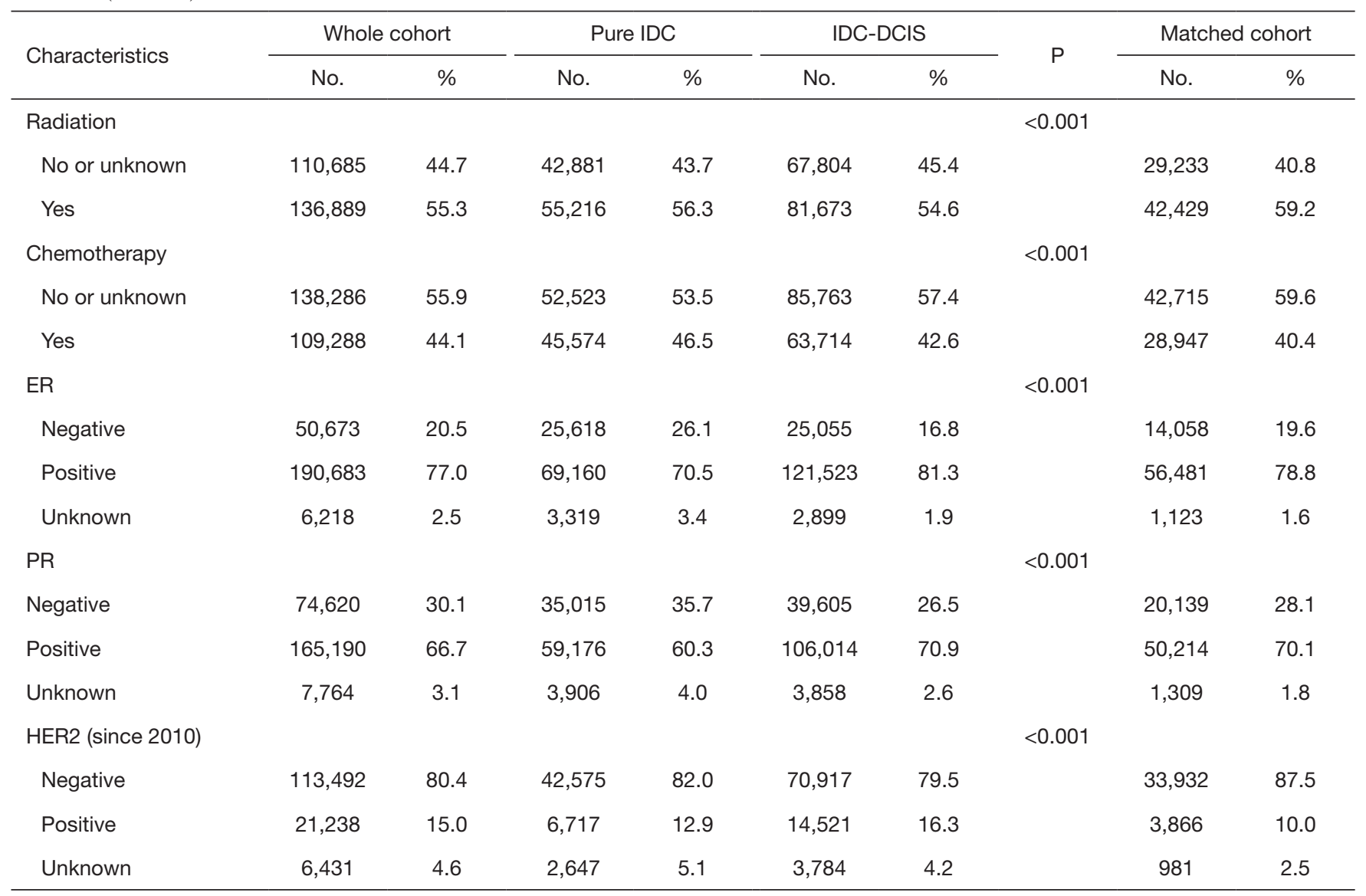

IDC, invasive ductal breast cancer; DCIS, ductal carcinoma in situ; BCS, breast conserving surgery.

ER negative cases, pure IDC had significantly or tendency of better survival outcomes in T1-T3 disease (BCSS: $\mathrm{P}=0.001$ for $\mathrm{T} 1, \mathrm{P}=0.002$ for $\mathrm{T} 2, \mathrm{P}=0.226$ for $\mathrm{T} 3$; OS: $\mathrm{P}=0.007$ for $\mathrm{T} 1, \mathrm{P}=0.077$ for $\mathrm{T} 2, \mathrm{P}=0.299$ for T3) (Figure 6), and in $\mathrm{N} 0-\mathrm{N} 3$ disease (BCSS: $\mathrm{P}<0.001$ for $\mathrm{N} 0, \mathrm{P}=0.342$ for $\mathrm{N} 1, \mathrm{P}=0.097$ for $\mathrm{N} 2, \mathrm{P}=0.673$ for $\mathrm{N} 3$; OS: $\mathrm{P}=0.013$ for $\mathrm{N} 0$, $\mathrm{P}=0.287$ for $\mathrm{N} 1, \mathrm{P}=0.134$ for $\mathrm{N} 2, \mathrm{P}=0.441$ for N3) (Figure 7)

According to multivariate COX analysis, existence of DCIS component was an independent unfavorable prognostic factor for ER negative cases (BCSS: $H R=1.152$, $\mathrm{P}<0.001$, 95\% CI: 1.081-1.228; OS: $\mathrm{HR}=1.089, \mathrm{P}=0.002$, 95\% CI: 1.033-1.148) (Table 2). However, existence of DCIS component was an independent favorable prognostic factor for ER positive cases (BCSS: $\mathrm{HR}=0.924, \mathrm{P}=0.006$, 95\% CI: 0.873-0.978; OS: $\mathrm{HR}=0.919, \mathrm{P}<0.001,95 \%$ CI: 0.888-0.951) (Table 3). For cases with HER2 status information diagnosed between 2010 and 2015, in spite of a relatively short follow up, existence of DCIS component was still an independent favorable prognostic factor in ER positive cases for BCSS (HR $=0.879,95 \%$ CI: $0.790-0.977$, $\mathrm{P}=0.017)$ and $\mathrm{OS}(\mathrm{HR}=0.931,95 \% \mathrm{CI}: 0.872-0.993$, $\mathrm{P}=0.031$ ).

\section{Discussion}

Our study was among the largest to address the issue of the prognostic significance of co-existence DCIS component in IDC not only based on the whole population-based cohort but also a matched case-control cohort. According to our study, there were great differences in the distributions of clinical-pathological characteristics between IDC-DCIS and pure IDC. Patients with IDC-DCIS had significantly better survival outcomes compared with those with pure IDC in the whole cohort. However, based on the matched cohort, IDC-DCIS had a similar BCSS but a higher OS compared with pure IDC. The existence of DCIS component was an 

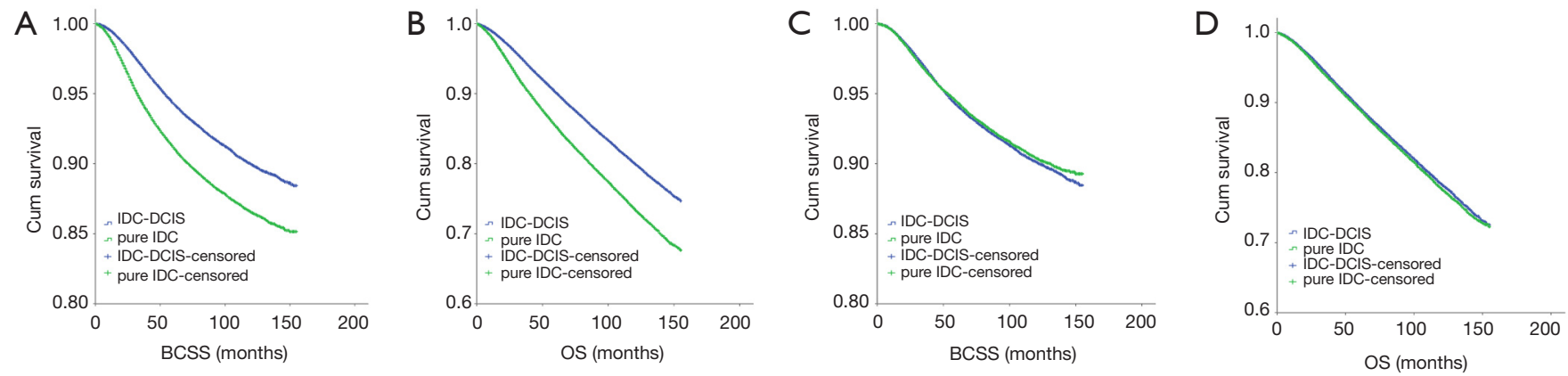

Figure 1 Kaplan-Meier survival curves among IDC-DCIS and pure IDC in the whole cohort and in the matched case-control cohort. (A) BCSS in the whole cohort; (B) OS in the whole cohort; (C) BCSS in the matched cohort; (D) OS in the matched cohort. IDC, invasive ductal breast cancer; DCIS, ductal carcinoma in situ; BCSS, breast cancer-specific survival; OS, overall survival.
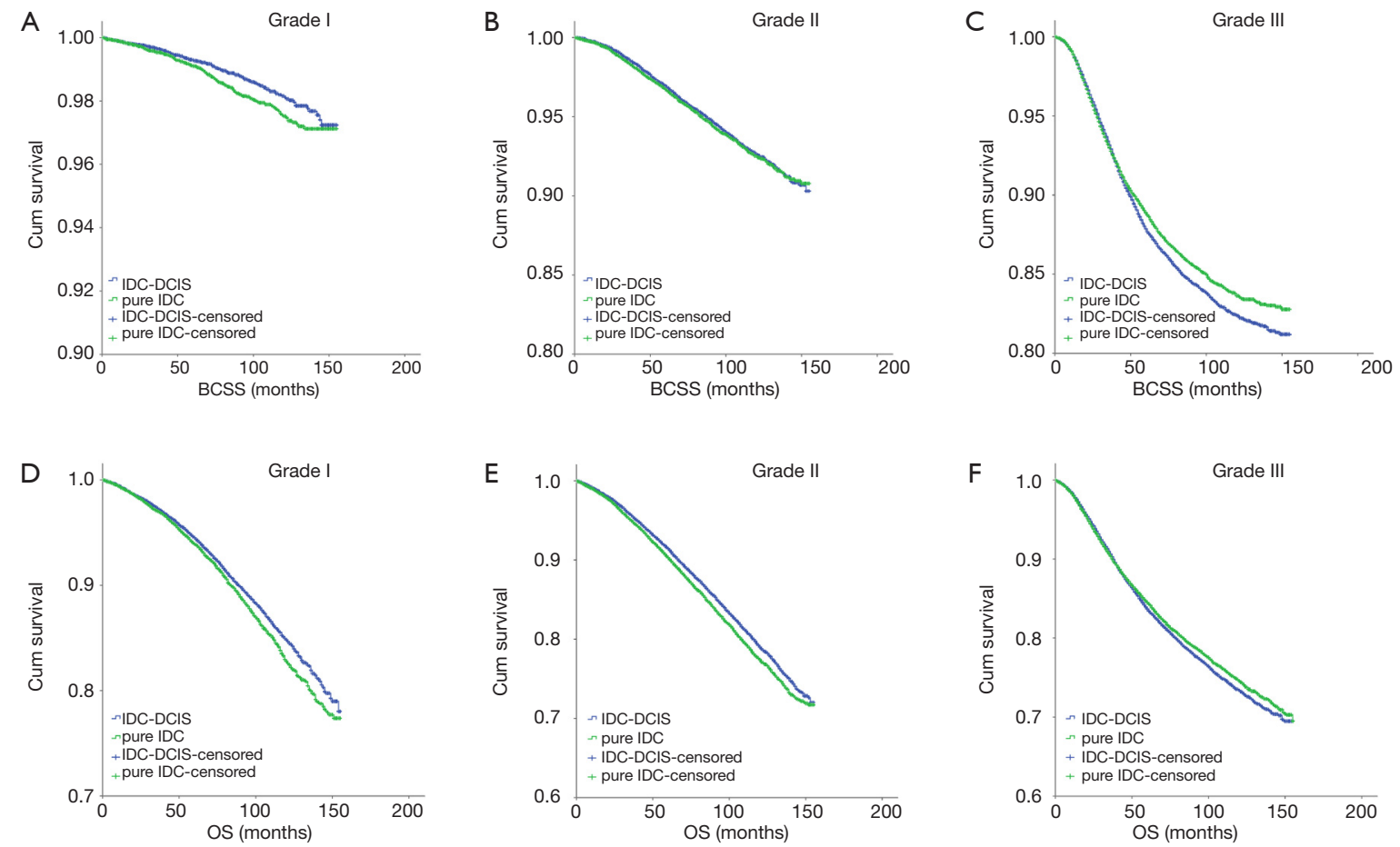

Figure 2 Kaplan-Meier survival curves among IDC-DCIS and pure IDC stratified by histologic grade in the matched case-control cohort. (A) BCSS in grade I; (B) BCSS in grade II; (C) BCSS in grade III; (D) OS in grade I; (E) OS in grade II; (F) OS in grade III. IDC, invasive ductal breast cancer; DCIS, ductal carcinoma in situ; BCSS, breast cancer-specific survival; OS, overall survival.

independent favorable prognostic factor for ER positive cases.

Some studies recognized that IDC-DCIS represents a clinical and biological entity distinct from pure IDC and showed that IDC-DCIS was associated with smaller tumor size, less lymph node involvement and well differentiated grade tumors $(8,9,11)$, which was in accord with the results of our study. There was still controversy regarding higher expression rate of ER and $\mathrm{PR}$ in IDCDCIS than in pure IDC $(1,4,8,12)$. Our study confirmed the higher positivity of ER and PR in IDC-DCIS based on a large sample size. Mylonas et al. found significantly increased HER2 amplification in pure IDC and assumed decreased tumor aggressiveness for IDC-DCIS (12). 

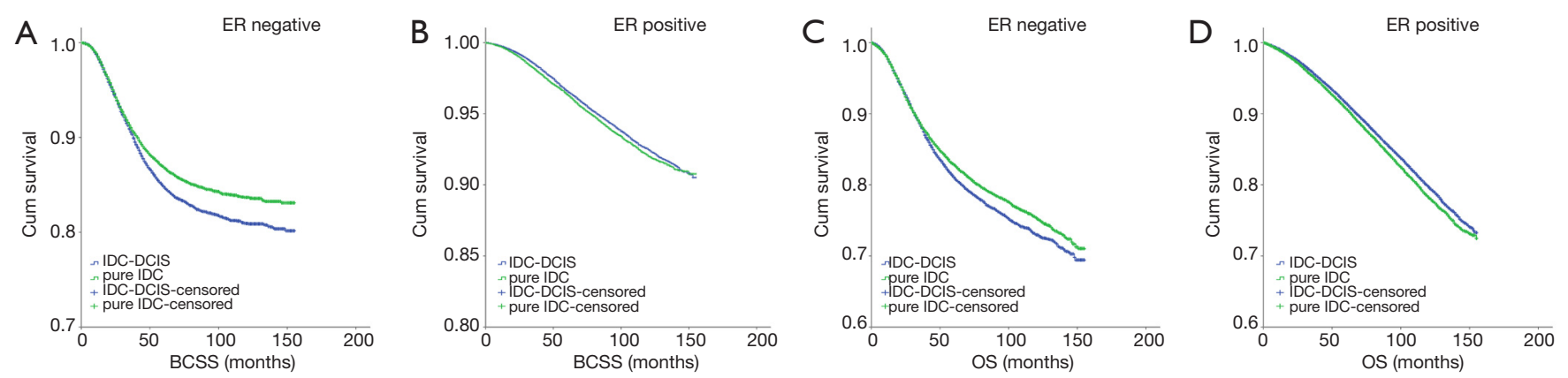

Figure 3 Kaplan-Meier survival curves among IDC-DCIS and pure IDC stratified by ER status in the matched case-control cohort. (A) BCSS in ER negative cases; (B) BCSS in ER positive cases; (C) OS in ER negative cases; (D) OS in ER positive cases. IDC, invasive ductal breast cancer; DCIS, ductal carcinoma in situ; BCSS, breast cancer-specific survival; OS, overall survival.
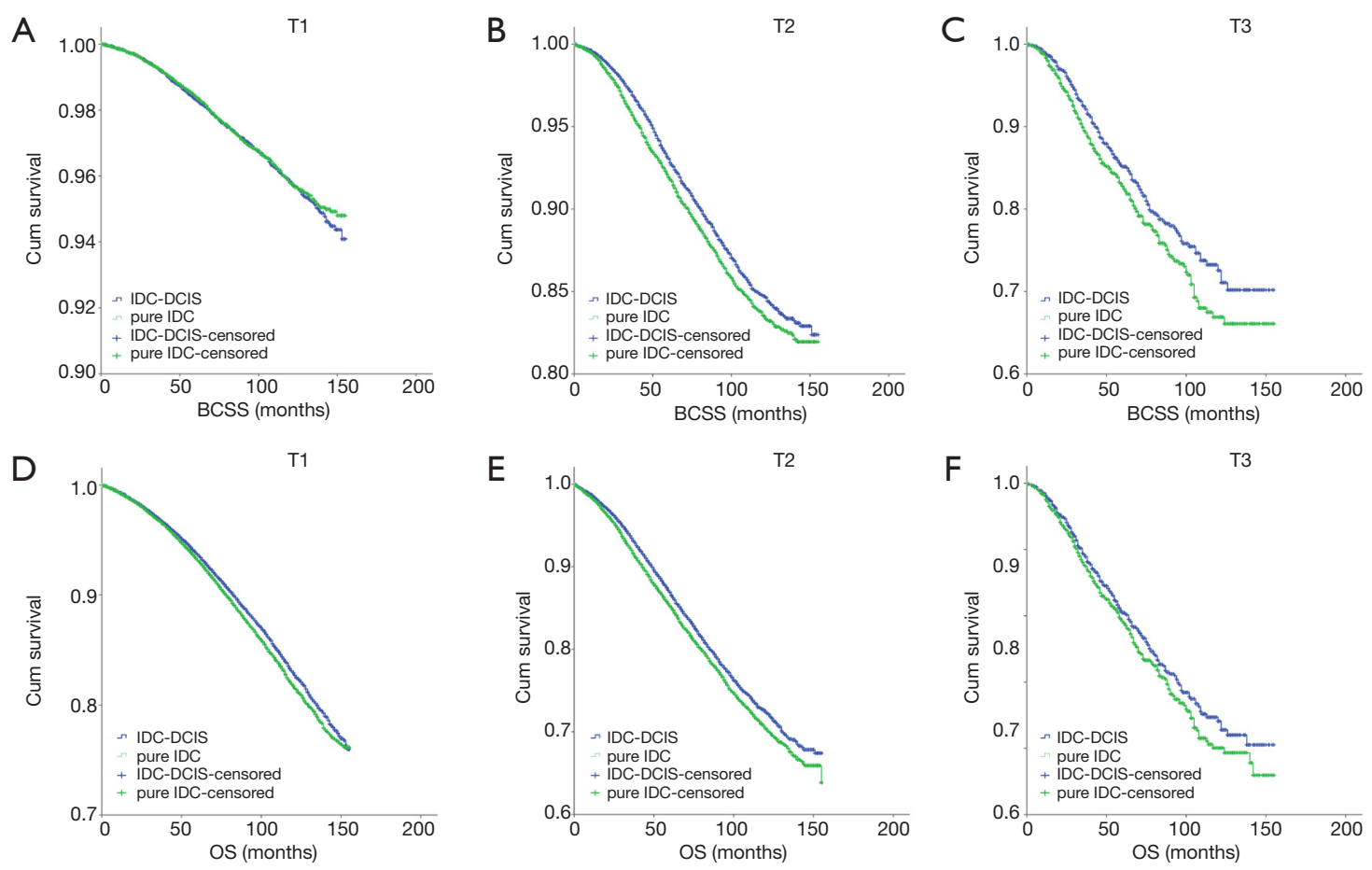

Figure 4 Kaplan-Meier survival curves among IDC-DCIS and pure IDC stratified by T category for ER positive cases in the matched casecontrol cohort. (A) BCSS in T1; (B) BCSS in T2; (C) BCSS in T3; (D) OS in T1; (E) OS in T2; (F) OS in T3. IDC, invasive ductal breast cancer; DCIS, ductal carcinoma in situ; BCSS, breast cancer-specific survival; OS, overall survival.

Although Papantoniou et al. suggested that IDC-DCIS was a more aggressive phenotype due to significantly higher Ki-67 expression compared with pure IDC (4), Wong et al. indicated that Ki-67 was lower in IDCDCIS than in size-adjusted pure IDC and predicted lower biological aggressiveness (10). Wong et al. also found that pure IDC cases were increasingly self-detected compared with IDC-DCIS lesions, which were detected by patient screening, implicating the advanced tumor stage and higher tumor aggressiveness of pure IDCs (8). These data corroborated our findings implying that patients with IDC-DCIS tended to present lower disease aggressiveness than pure IDC. Cases with T1mic were excluded in our study as Lillemoe et al. suggested that the 

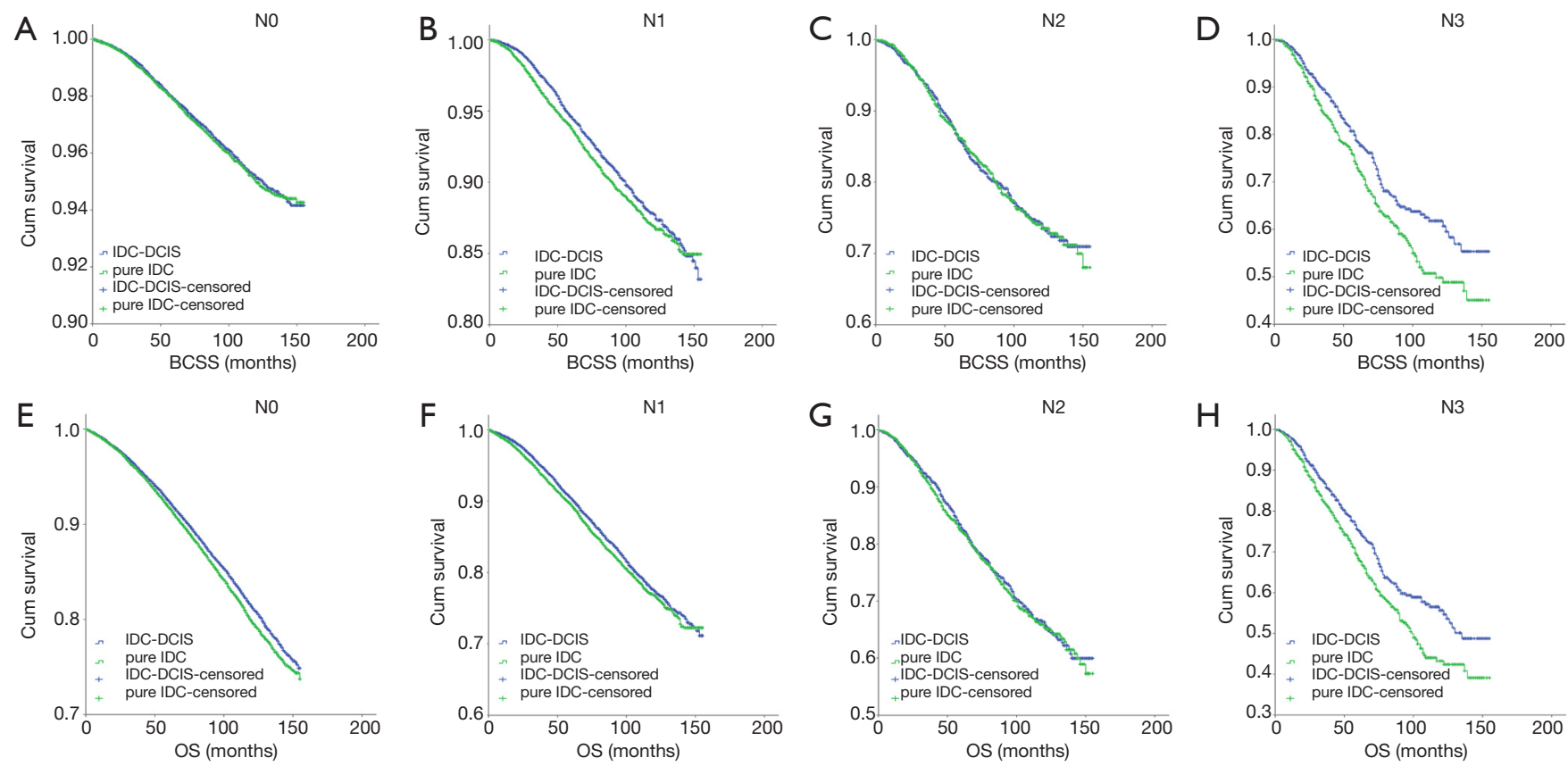

Figure 5 Kaplan-Meier survival curves among IDC-DCIS and pure IDC stratified by $\mathrm{N}$ category for ER positive cases in the matched casecontrol cohort. (A) BCSS in N0; (B) BCSS in N1; (C) BCSS in N2; (D) BCSS in N3; (E) OS in N0; (F) OS in N1; (G) OS in N2; (H) OS in N3. IDC, invasive ductal breast cancer; DCIS, ductal carcinoma in situ; BCSS, breast cancer-specific survival; OS, overall survival.
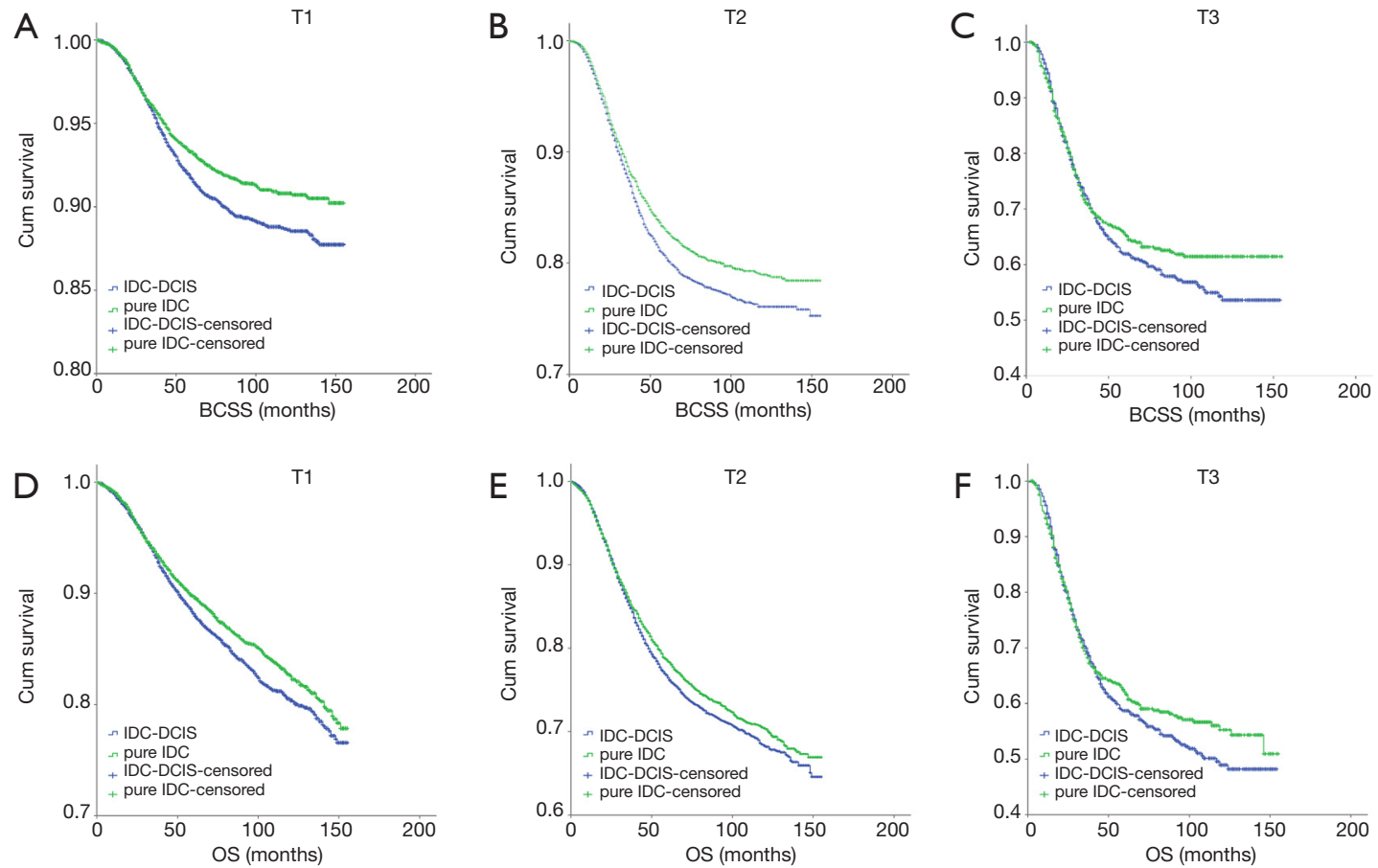

Figure 6 Kaplan-Meier survival curves among IDC-DCIS and pure IDC stratified by T category for ER negative cases in the matched casecontrol cohort. (A) BCSS in T1; (B) BCSS in T2; (C) BCSS in T3; (D) OS in T1; (E) OS in T2; (F) OS in T3. IDC, invasive ductal breast cancer; DCIS, ductal carcinoma in situ; BCSS, breast cancer-specific survival; OS, overall survival. 

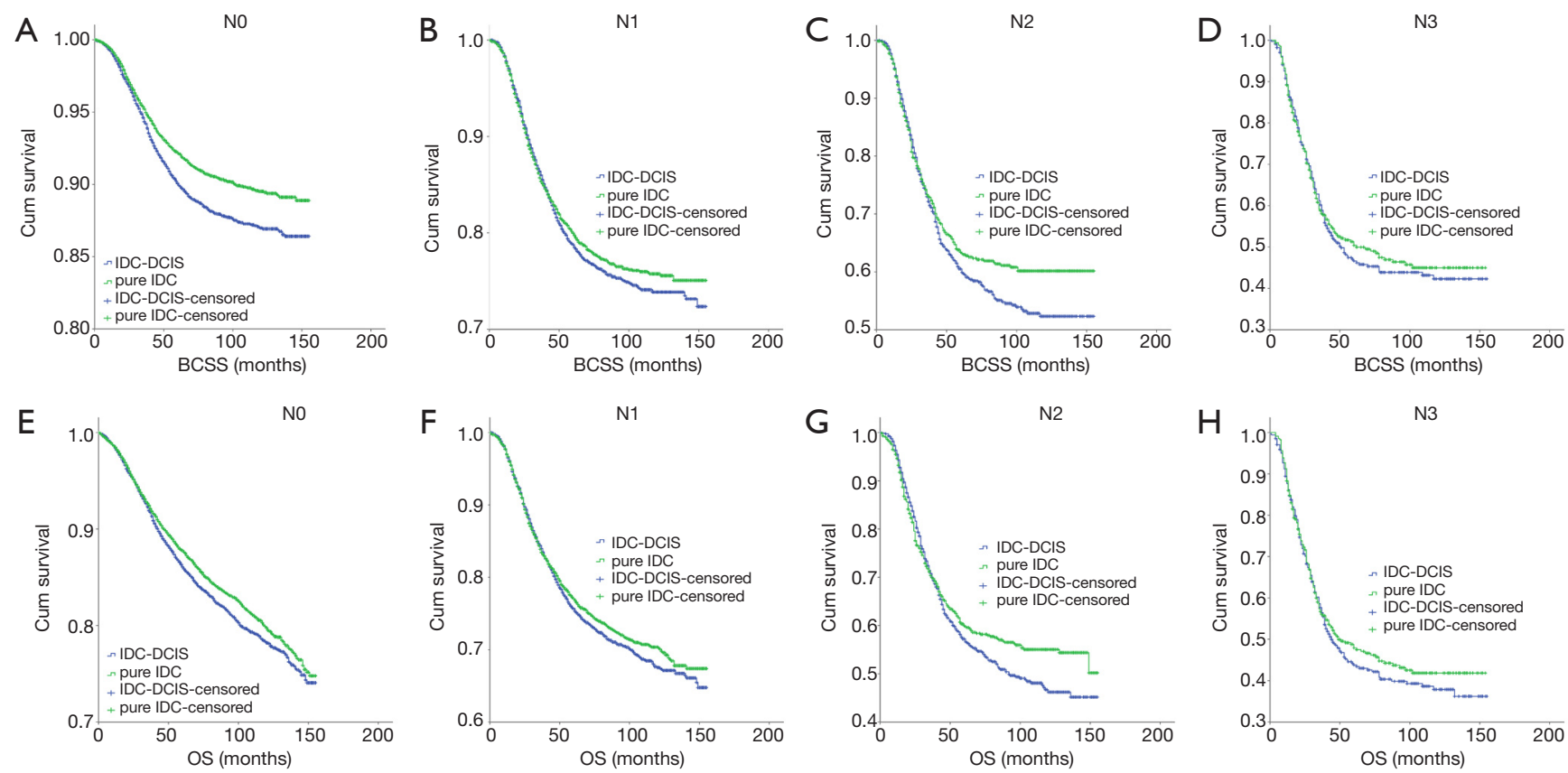

Figure 7 Kaplan-Meier survival curves among IDC-DCIS and pure IDC stratified by N category for ER negative cases in the matched casecontrol cohort. (A) BCSS in N0; (B) BCSS in N1; (C) BCSS in N2; (D) BCSS in N3; (E) OS in N0; (F) OS in N1; (G) OS in N2; (H) OS in N3. IDC, invasive ductal breast cancer; DCIS, ductal carcinoma in situ; BCSS, breast cancer-specific survival; OS, overall survival.

T1mic breast cancer had similar clinical behavior to DCIS and could be treated and followed up as pure DCIS $(13,14)$.

According to our study BCS rate was significantly lower in IDC-DCIS compared with pure IDC. Wong $\mathrm{H}$ et al. also found IDC-DCIS was less likely to be treated with BCS (8). A number of researchers found that co-existence DCIS was associated with positive margins, suggesting a potentially higher local recurrence (LR) rate $(5,15,16)$. Kim et al. indicated that the incidence of LR was higher in IDC-DCIS than in pure IDC patients (8.9\% vs. 6.3\%) (17). However, Mechera $e t a l$. found that the greater risk of LR in IDCDCIS was no longer significant on multivariate analysis (16), and Dieterich $e t a l$. showed that IDC-DCIS was associated with significantly lower rate of $\mathrm{LR}(\mathrm{P}=0.012)(11)$. Since the publication of the guideline on margins for BCS (18), $2 \mathrm{~mm}$ is adequate and DCIS component should not be a main obstacle for BCS in most cases.

A number of studies seemed to show a trend towards improved survival outcome in IDC-DCIS compared with pure IDC $(1,2,6,8,9,19)$. Logullo et al. found the concomitant DCIS component to be a predictor of improved survival independent of tumor size, but their study did not control for age or tumor grade (6). Furthermore, Matsukuma et al. analyzed survival outcomes without controlling for treatment variables (19). Wong et al. did not find difference in survival outcome between IDC-DCIS and pure IDC with a median follow up of 29.3 months (8). Chagpar et al. reported that the presence of DCIS was not an independent predictor of improved survival outcome on multivariate analysis (9). As these studies enrolled only limited cases and had a relatively short follow-up period, it was hard to confirm co-existence DCIS as an independent prognostic factor. Our study had the largest sample size to date and demonstrated that existence of DCIS component in IDC was an independent favorable prognostic factor for both BCSS and OS on multivariate analysis based on the whole population cohort. There could be a bias from 'screen detection', that is, IDC-DCIS group is more likely to be screen detected because of calcifying DCIS, which might be an independent prognostic indicator. Besides, the baseline of most important clinical-pathological characteristics was not balanced between the two cohorts. As a result, a further matched case-control analysis was conducted. Based on the matched cohort, co-existence DCIS was no longer an independent prognostic factor for BCSS but a favorable prognostic factor for OS.

Co-existence DCIS demonstrated quite different prognostic significance among ER positive and ER negative 
Table 2 Multivariate analysis of prognostic factors among ER negative patients with pure IDC and IDC-DCIS

\begin{tabular}{|c|c|c|c|c|c|c|}
\hline Characteristics & \multicolumn{3}{|c|}{ BCSS } & \multicolumn{3}{|c|}{ OS } \\
\hline Year of diagnosis & 0.987 & $0.976-0.998$ & 0.023 & 0.987 & $0.977-0.997$ & 0.010 \\
\hline \multicolumn{7}{|l|}{ Age } \\
\hline$\leq 60$ vs. $>60$ years & 0.729 & $0.681-0.780$ & $<0.001$ & 0.509 & $0.481-0.539$ & $<0.001$ \\
\hline Black vs. White & 1.306 & $1.203-1.418$ & $<0.001$ & 1.241 & $1.156-1.333$ & $<0.001$ \\
\hline Asian or Indian vs. White & 0.708 & $0.593-0.846$ & 0.001 & 0.653 & $0.559-0.764$ & $<0.001$ \\
\hline Histologic grade & & & $<0.001$ & & & 0.047 \\
\hline I vs. III & 0.330 & $0.157-0.695$ & 0.004 & 0.675 & $0.472-0.964$ & 0.030 \\
\hline T1 vs. T4 & 0.216 & $0.185-0.253$ & $<0.001$ & 0.234 & $0.203-0.269$ & $<0.001$ \\
\hline T2 vs. T4 & 0.435 & $0.377-0.502$ & $<0.001$ & 0.436 & $0.382-0.498$ & $<0.001$ \\
\hline T3 vs. T4 & 0.742 & $0.633-0.870$ & $<0.001$ & 0.743 & $0.641-0.862$ & $<0.001$ \\
\hline $\mathrm{N}$ stage & & & $<0.001$ & & & $<0.001$ \\
\hline N0 vs. N3 & 0.195 & $0.171-0.222$ & $<0.001$ & 0.244 & $0.216-0.275$ & $<0.001$ \\
\hline N1 vs. N3 & 0.411 & $0.363-0.466$ & $<0.001$ & 0.435 & $0.387-0.489$ & $<0.001$ \\
\hline N2 vs. N3 & 0.720 & $0.627-0.826$ & $<0.001$ & 0.757 & $0.665-0.862$ & $<0.001$ \\
\hline \multicolumn{7}{|c|}{ Existence of DCIS component } \\
\hline \multicolumn{7}{|l|}{ Chemotherapy } \\
\hline Yes vs. none or unknown & 0.610 & $0.561-0.664$ & $<0.001$ & 0.453 & $0.425-0.483$ & $<0.001$ \\
\hline \multicolumn{7}{|l|}{ PR status } \\
\hline Positive vs. negative & 0.744 & $0.573-0.968$ & 0.027 & 0.794 & $0.645-0.978$ & 0.030 \\
\hline
\end{tabular}

IDC, invasive ductal breast cancer; DCIS, ductal carcinoma in situ; BCSS, breast cancer-specific survival; OS, overall survival; HR, hazard ratio; $\mathrm{Cl}$, confidence interval.

disease, which was an important finding in our study. ER positive cases accounted for $97.9 \%$ and $92.1 \%$ in grade I and II disease while ER negative cases accounted for $47.4 \%$ in grade III disease. As a result, IDC-DCIS showed better survival outcomes in grade I and II disease, while pure IDC showed improved survivals in grade III disease according to the subgroup analysis.
Traditionally, $\mathrm{T}$ and $\mathrm{N}$ category indicated the status of cancer progression. According to the subgroup analysis, among ER positive cases, IDC-DCIS showed improved survival outcomes compared with pure IDC in T1-T3 and N0-N3 categories. Specifically IDC-DCIS showed a similar BCSS with pure IDC in T1 and N0 categories probably due to the scarce cancer-related death events in 
Table 3 Multivariate analysis of prognostic factors among ER positive patients with pure IDC and IDC-DCIS

\begin{tabular}{|c|c|c|c|c|c|c|}
\hline Characteristics & \multicolumn{3}{|c|}{ BCSS } & \multicolumn{3}{|c|}{ os } \\
\hline Year of diagnosis & 0.978 & $0.967-0.990$ & $<0.001$ & 0.968 & $0.961-0.974$ & $<0.001$ \\
\hline \multicolumn{7}{|l|}{ Age } \\
\hline$\leq 60$ vs. $>60$ years & 0.668 & $0.627-0.711$ & $<0.001$ & 0.312 & $0.299-0.326$ & $<0.001$ \\
\hline Black vs. White & 1.375 & $1.244-1.519$ & $<0.001$ & 1.326 & $1.241-1.416$ & $<0.001$ \\
\hline Asian or Indian vs. White & 0.746 & $0.646-0.861$ & $<0.001$ & 0.666 & $0.607-0.731$ & $<0.001$ \\
\hline Histologic grade & & & $<0.001$ & & & $<0.001$ \\
\hline I vs. III & 0.234 & $0.208-0.264$ & $<0.001$ & 0.556 & $0.527-0.586$ & $<0.001$ \\
\hline $\mathrm{T} 1$ vs. T4 & 0.185 & $0.158-0.216$ & $<0.001$ & 0.226 & $0.201-0.255$ & $<0.001$ \\
\hline T2 vs. T4 & 0.433 & $0.374-0.501$ & $<0.001$ & 0.402 & $0.357-0.452$ & $<0.001$ \\
\hline T3 vs. T4 & 0.699 & $0.593-0.824$ & $<0.001$ & 0.653 & $0.569-0.750$ & $<0.001$ \\
\hline $\mathrm{N}$ stage & & & $<0.001$ & & & $<0.001$ \\
\hline No vs. N3 & 0.192 & $0.168-0.218$ & $<0.001$ & 0.258 & $0.232-0.288$ & $<0.001$ \\
\hline N1 vs. N3 & 0.354 & $0.313-0.400$ & $<0.001$ & 0.351 & $0.315-0.391$ & $<0.001$ \\
\hline N2 vs. N3 & 0.596 & $0.522-0.681$ & $<0.001$ & 0.596 & $0.529-0.671$ & $<0.001$ \\
\hline \multicolumn{7}{|l|}{ Existence of DCIS component } \\
\hline \multicolumn{7}{|l|}{ Radiation } \\
\hline \multicolumn{7}{|l|}{ Chemotherapy } \\
\hline Yes vs. none or unknown & 0.794 & $0.738-0.853$ & $<0.001$ & 0.533 & $0.508-0.559$ & $<0.001$ \\
\hline \multicolumn{7}{|l|}{ PR status } \\
\hline Positive vs. negative & 0.653 & $0.607-0.702$ & $<0.001$ & 0.813 & $0.775-0.853$ & $<0.001$ \\
\hline
\end{tabular}

IDC, invasive ductal breast cancer; DCIS, ductal carcinoma in situ; BCSS, breast cancer-specific survival; OS, overall survival; HR, hazard ratio; $\mathrm{Cl}$, confidence interval.

these cases. IDC-DCIS showed only tendency of improved survivals in $\mathrm{N} 2$ cases probably due to the relatively small sample size and less death events for N2 category and longer follow up was further needed. Current evidence demonstrated that DCIS component in IDC-DCIS showed similar immunohistochemical marker (20-22) and genomic profiles (23-26) to its invasive component. Cells from the DCIS component of lesions with co-existing IDC exhibited a substantial number of molecular alterations compared with those expressed in pure DCIS preceding apparent morphological progression to invasive cancer $(23,25)$. These results suggest DCIS is frequently a precursor lesion 
for co-existing IDC and IDC in IDC-DCIS tends to evolve as a result of a more incremental accumulation of milder suppressor gene defects, whereas in patients with pure IDC, IDC is postulated to arise de novo as a result of one or more drastic tumor suppressor gene defects $(8,22)$. The findings of the subgroup analysis in ER positive cases supported the hypothesis of linear progression model to explain the progression from DCIS to IDC $(25,27-31)$ in which the presence of DCIS in conjunction with invasive cancer could be considered as an earlier stage of disease and thus having a better prognosis.

Furthermore, among ER positive cases, IDC-DCIS showed a better OS in T1 and N0 in spite of a similar BCSS. There might be something related with tumor immunity behind it. The presence of DCIS was associated with a change in the tumor microenvironment, playing an important role in tumor progression $(32,33)$. It was reported that $81 \%$ of DCIS lesions contained PD-L1+ tumor infiltrating lymphocytes (34). This cell-mediated immunological response might lead to an improved prognosis for patients with concomitant DCIS (35). Further research for mechanisms is worthwhile.

However, among ER negative cases, co-existence DCIS had a negative impact on survival. Pure IDC had improved survivals compared with IDC-DCIS in T1-T3 and N0N3 categories. In some subgroups, pure IDC showed only tendency of improved survival probably due to the relatively small sample size but the trend was apparent. The opposite trend for survival benefit in ER negative disease indicated that different mechanisms behind the progression of DCIS to IDC in different ER status might be involved and further research in this aspect is worthwhile.

The strength of our study lay in its homogeneous study population and the statistical significance confirmed by multivariate analyses in whole cohort, matched cohort, and various subgroup analyses. However, there were still some limitations to be pointed out. Most importantly, the data regarding the grade or extent of DCIS was unavailable. Kim et al. found that patients with IDC accompanying highgrade DCIS had a 2.5 -fold higher probability of local or distant relapse than did those with IDC accompanying lowgrade DCIS (17). However, that study also revealed that IDC with non-high-grade DCIS has a tendency to be of low histologic grade and that IDC with high-grade DCIS tends to be of high histologic grade (17). No differences were observed between the grade of the in situ and the invasive component of infiltrating carcinomas (36). After all, further work is warranted to determine the effect of extent and grade of DCIS on prognosis in patients with invasive cancer. Besides, the retrospective study design had intrinsic defects and information of HER2 status before 2010 and endocrine therapy was also unavailable and could not be evaluated.

In conclusion, IDC-DCIS had significantly better survival outcomes than pure IDC probably due to the less aggressive characteristics. In the matched casecontrol analysis, co-existence of DCIS was an independent favorable prognostic factor in ER positive cases, but it was an independent negative prognostic factor in ER negative cases. The survival trend was consistent for cases in most $\mathrm{T}$ and $\mathrm{N}$ categories. Co-existing DCIS could have significant prognostic value and implications for adjuvant treatment decision-making process in these cases. The mechanisms behind it warrant further research.

\section{Acknowledgments}

None.

\section{Footnote}

Conflicts of Interest: The authors have no conflicts of interest to declare.

Ethical Statement: The authors are accountable for all aspects of the work in ensuring that questions related to the accuracy or integrity of any part of the work are appropriately investigated and resolved. Informed consent was not required because personal identifying information was not involved. This study was reviewed and approved by the Institutional Review Board of Obstetrics and Gynecology Hospital of Fudan University.

\section{References}

1. Jo BH, Chun YK. Heterogeneity of invasive ductal carcinoma: proposal for a hypothetical classification. J Korean Med Sci 2006;21:460-8.

2. Carabias-Meseguer P, Zapardiel I, Cusido-Gimferrer M, et al. Influence of the in situ component in 389 infiltrating ductal breast carcinomas. Breast Cancer 2013;20:213-7.

3. Pinder SE. Ductal carcinoma in situ (DCIS): pathological features, differential diagnosis, prognostic factors and specimen evaluation. Mod Pathol 2010;23 Suppl 2:S8-13.

4. Papantoniou V, Sotiropoulou E, Valsamaki P, et al. Breast density, scintimammographic $(99 \mathrm{~m}) \mathrm{Tc}(\mathrm{V}) \mathrm{DMSA}$ uptake, 
and calcitonin gene related peptide (CGRP) expression in mixed invasive ductal associated with extensive in situ ductal carcinoma (IDC + DCIS) and pure invasive ductal carcinoma (IDC): correlation with estrogen receptor (ER) status, proliferation index $\mathrm{Ki}-67$, and histological grade. Breast Cancer 2011;18:286-91.

5. Dzierzanowski M, Melville KA, Barnes PJ, et al. Ductal carcinoma in situ in core biopsies containing invasive breast cancer: correlation with extensive intraductal component and lumpectomy margins. J Surg Oncol 2005;90:71-6.

6. Logullo AF, Godoy AB, Mourao-Neto M, et al. Presence of ductal carcinoma in situ confers an improved prognosis for patients with T1N0M0 invasive breast carcinoma. Braz J Med Biol Res 2002;35:913-9.

7. Yu KD, Wu LM, Liu GY, et al. Different distribution of breast cancer subtypes in breast ductal carcinoma in situ (DCIS), DCIS with microinvasion, and DCIS with invasion component. Ann Surg Oncol 2011;18:1342-8.

8. Wong H, Lau S, Yau T, et al. Presence of an in situ component is associated with reduced biological aggressiveness of size-matched invasive breast cancer. Br J Cancer 2010;102:1391-6.

9. Chagpar AB, McMasters KM, Sahoo S, et al. Does ductal carcinoma in situ accompanying invasive carcinoma affect prognosis? Surgery 2009;146:561-7; discussion 567-8.

10. Wong H, Lau S, Leung R, et al. Coexisting ductal carcinoma in situ independently predicts lower tumor aggressiveness in node-positive luminal breast cancer. Med Oncol 2012;29:1536-42.

11. Dieterich M, Hartwig F, Stubert J, et al. Accompanying DCIS in breast cancer patients with invasive ductal carcinoma is predictive of improved local recurrence-free survival. Breast 2014;23:346-51.

12. Mylonas I, Makovitzky J, Jeschke U, et al. Expression of Her2/neu, steroid receptors (ER and PR), Ki67 and p53 in invasive mammary ductal carcinoma associated with ductal carcinoma In Situ (DCIS) Versus invasive breast cancer alone. Anticancer Res 2005;25:1719-23.

13. Lillemoe TJ, Tsai ML, Swenson KK. Clinicopathologic analysis of a large series of microinvasive breast cancers. breast J 2018;24:574-9.

14. Kim M, Kim HJ, Chung YR, et al. Microinvasive Carcinoma versus Ductal Carcinoma In Situ: A Comparison of Clinicopathological Features and Clinical Outcomes. J Breast Cancer 2018;21:197-205.

15. Kurniawan ED, Wong MH, Windle I, et al. Predictors of surgical margin status in breast-conserving surgery within a breast screening program. Ann Surg Oncol 2008;15:2542-9.

16. Mechera R, Viehl CT, Oertli D. Factors predicting inbreast tumor recurrence after breast-conserving surgery. Breast Cancer Res Treat 2009;116:171-7.

17. Kim JY, Han W, Moon HG, et al. Grade of ductal carcinoma in situ accompanying infiltrating ductal carcinoma as an independent prognostic factor. Clin Breast Cancer 2013;13:385-91.

18. Morrow M, Van Zee KJ, Solin LJ, et al. Society of Surgical Oncology-American Society for Radiation OncologyAmerican Society of Clinical Oncology Consensus Guideline on Margins for Breast-Conserving Surgery with Whole-Breast Irradiation in Ductal Carcinoma In Situ. Pract Radiat Oncol 2016;6:287-95.

19. Matsukuma A, Enjoji M, Toyoshima S. Ductal carcinoma of the breast. An analysis of proportions of intraductal and invasive components. Pathol Res Pract 1991;187:62-7.

20. Leong AS, Sormunen RT, Vinyuvat S, et al. Biologic markers in ductal carcinoma in situ and concurrent infiltrating carcinoma. A comparison of eight contemporary grading systems. Am J Clin Pathol 2001;115:709-18.

21. Wärnberg F, Nordgren H, Bergkvist L, et al. Tumour markers in breast carcinoma correlate with grade rather than with invasiveness. Br J Cancer 2001;85:869-74.

22. Steinman S, Wang J, Bourne P, et al. Expression of cytokeratin markers, ER-alpha, PR, HER-2/neu, and EGFR in pure ductal carcinoma in situ (DCIS) and DCIS with co-existing invasive ductal carcinoma (IDC) of the breast. Ann Clin Lab Sci 2007;37:127-34.

23. Iakovlev VV, Arneson NC, Wong V, et al. Genomic differences between pure ductal carcinoma in situ of the breast and that associated with invasive disease: a calibrated aCGH study. Clin Cancer Res 2008;14:4446-54.

24. Aubele M, Mattis A, Zitzelsberger H, et al. Extensive ductal carcinoma In situ with small foci of invasive ductal carcinoma: evidence of genetic resemblance by CGH. Int J Cancer 2000;85:82-6.

25. Castro NP, Osorio CA, Torres C, et al. Evidence that molecular changes in cells occur before morphological alterations during the progression of breast ductal carcinoma. Breast Cancer Res 2008;10:R87.

26. Johnson CE, Gorringe KL, Thompson ER, et al. Identification of copy number alterations associated with the progression of DCIS to invasive ductal carcinoma. Breast Cancer Res Treat 2012;133:889-98.

27. Lee S, Stewart S, Nagtegaal I, et al. Differentially expressed genes regulating the progression of ductal 
Page 14 of 14

carcinoma in situ to invasive breast cancer. Cancer Res 2012;72:4574-86.

28. Kuerer HM, Albarracin CT, Yang WT, et al. Ductal carcinoma in situ: state of the science and roadmap to advance the field. J Clin Oncol 2009;27:279-88.

29. Wiechmann L, Kuerer HM. The molecular journey from ductal carcinoma in situ to invasive breast cancer. Cancer 2008;112:2130-42.

30. Singletary SE. A working model for the time sequence of genetic changes in breast tumorigenesis. J Am Coll Surg 2002;194:202-16.

31. Dalgin GS, Alexe G, Scanfeld D, et al. Portraits of breast cancer progression. BMC Bioinformatics 2007;8:291.

32. Barsky SH, Karlin NJ. Mechanisms of disease: breast tumor pathogenesis and the role of the myoepithelial cell. Nat Clin Pract Oncol 2006;3:138-51.

Cite this article as: Chen $\mathrm{H}$, Bai F, Wang $M$, Zhang $M$, Zhang $\mathrm{P}, \mathrm{Wu} \mathrm{K}$. The prognostic significance of co-existence ductal carcinoma in situ in invasive ductal breast cancer: a large population-based study and a matched case-control analysis. Ann Transl Med 2019;7(18):484. doi: 10.21037/atm.2019.08.16

\section{Chen et al. Prognostic significance of co-existence DCIS in IDC}

33. Sharma M, Beck AH, Webster JA, et al. Analysis of stromal signatures in the tumor microenvironment of ductal carcinoma in situ. Breast Cancer Res Treat 2010;123:397-404.

34. Thompson E, Taube JM, Elwood H, et al. The immune microenvironment of breast ductal carcinoma in situ. Mod Pathol 2016;29:249-58.

35. Black MM, Zachrau RE, Hankey BF, et al. Prognostic significance of in situ carcinoma associated with invasive breast carcinoma. A natural experiment in cancer immunology? Cancer 1996;78:778-88.

36. Giardina C, Serio G, Lepore G, et al. Pure ductal carcinoma in situ and in situ component of ductal invasive carcinoma of the breast. A preliminary morphometric study. J Exp Clin Cancer Res 2003;22:279-88. 